\title{
Bacterial diversity associated with the abdomens of naturally Plasmodium-infected and non-infected Nyssorhynchus darlingi
}

\author{
Tatiane Marques Porangaba Oliveira ${ }^{*^{*}}$, Sabri Saeed Sanabani ${ }^{2}$ and Maria Anice Mureb Sallum ${ }^{*}$
}

\begin{abstract}
Background: The bacterial community present in the abdomen in Anophelinae mosquitoes can influence mosquito susceptibility to Plasmodium infection. Little is known about the bacteria associated with Nyssorhynchus darlingi, a primary malaria vector in the Amazon basin. We investigated the abdominal bacterial community compositions of naturally Plasmodium-infected (P-positive, $n=9$ ) and non-infected ( $P$-negative, $n=7$ ) $\mathrm{Ny}$. darlingi from the Brazilian Amazon region through massive parallel sequencing of the bacterial $V 4$ variable region of the $16 \mathrm{~S}$ rRNA gene.

Results: Bacterial richness of Ny. darlingi encompassed 379 operational taxonomic units (OTUs), the majority of them belonging to the Proteobacteria, Firmicutes and Bacteroides phyla. Escherichia/Shigella and Pseudomonas were more abundant in the P-positive and P-negative groups, respectively, than in the opposite groups. Enterobacter was found only in the $P$-negative group. The results of statistical analyses conducted to compare bacterial abundance and diversity between Plasmodium-infected and Plasmodium-non-infected mosquitoes were not significant.

Conclusions: This study increased knowledge about bacterial composition in Ny. darlingi and revealed that Plasmodium-positive and Plasmodium-negative groups share a common core of bacteria. The genera Prevotella 9, Sphingomonas, Bacteroides, and Bacillus were reported for the first time in Ny. darlingi.
\end{abstract}

Keywords: Pseudomonas, Escherichia/Shigella, Malaria, Ny. darlingi, Amazon, Plasmodium

\section{Background}

Malaria is one of the world's most common and deadly tropical diseases. Recent data from the World Health Organization (WHO) has estimated that there were 219 million clinical cases and 435,000 estimated deaths in 87 malaria-endemic countries with ongoing malaria transmission in 2017 [1]. Brazil, Nicaragua and Venezuela registered increased malaria incidences in 2017. Malaria infections in Brazil are predominantly caused by Plasmodium vivax, accounting for $>84 \%$ of cases [2]. Most

\footnotetext{
*Correspondence: porangaba@usp.br; masallum@usp.br

'Departamento de Epidemiologia, Faculdade de Saúde Pública, Universidade de São Paulo, São Paulo, SP, Brazil

Full list of author information is available at the end of the article
}

malaria cases occur in the Amazon region, where the primary vector is Nyssorhynchus darlingi.

It is known that the mosquito midgut microbiota can affect the development of Plasmodium parasites in mosquitoes [3-8]. Isolates of Enterobacter amnigenus and Enterobacter cloacae are able to impair the sporogonic development of $P$. vivax, while Serratia marcescens completely inhibits $P$. vivax oocyst development in Anopheles albimanus [9]. One Enterobacter sp. strain (Esp_Z) isolated from the gut of wild Anopheles arabiensis confers resistance to $P$. falciparum infection in Anopheles gambiae [3]. Other studies have reported that Serratia marcescens blocks the development of P. falciparum ookinetes in An. gambiae [4, 10]. It has been proposed that bacteria present in the midgut modulate 
Plasmodium infection in the mosquito through at least two mechanisms: production of bacterial metabolites that impair the development of the parasite and induction of the immune response [3, 10-12].

Following an infective blood meal, the numbers of ookinetes present in the midgut lumens of aseptic and septic An. gambiae are similar, while the numbers of ookinetes present in the midgut epithelia of aseptic mosquitoes are 2.5 times higher than those in septic mosquitoes, suggesting that the bacterial effect occurs during ookinete development and invasion of the midgut epithelium [11]. Additionally, Anopheles and Aedes mosquitoes that are fed antibiotics to reduce microbiota populations have higher rates of Plasmodium and dengue virus infection than untreated mosquitoes [13]. Thus, symbiotic bacteria can be an alternative tool for blocking Plasmodium development in mosquitoes by decreasing vector competence.

The interaction between insects and microbes has been studied in a variety of vector species; however, little is known about the bacterial communities associated with the abdomen in the primary neotropical vector, $N y$. darlingi [14-16]. This study compared the bacterial communities associated with the abdomens of noninfected and Plasmodium-infected field-collected females of $\mathrm{Ny}$. darlingi.

\section{Results}

\section{Sequencing data output}

Twenty-four female abdomens were employed to generate bacterial community data. Sixteen samples (66.6\%) were successfully amplified and sequenced for the 250 bp $16 \mathrm{~S}$ rRNA genomic region. Nine were infected with Plasmodium (3 from Cruzeiro do Sul, 1 from Mâncio Lima, 1 from Lábrea, and 4 from Machadinho D'Oeste), and seven were not infected with Plasmodium (6 from Cruzeiro do Sul and 1 from Machadinho D'Oeste) (Additional file 1).

The MiSeq Illumina platform generated 2,505,232 raw reads ( $\mathrm{R} 1$ and $\mathrm{R} 2$ ) from 16 abdomens, with a median of 135,829 reads (range: $28,430-434,102$ ). The read values were 1,379,570 for the $P$-positive samples and 1,125,662 for the $P$-negative samples. After assembly and filtering of the OTUs with less than 5 sequences, 275,203 sequences were retained. These sequences were assigned to 407 unique OTUs (Additional file 2); 96 were found only in the $P$-positive group, 172 were found only in the $P$-negative group, and 139 were identified in both groups.

\section{Bacterial compositions}

Twenty-eight OTUs corresponded to chloroplasts, mitochondria, and unassigned OTUs. Of the 379 bacterial OTUs obtained, 333 were identified to the genus level.
Additionally, 35 OTUs could only be identified at the family level, 4 OTUs could only be identified at the order level, 6 could only be identified at the class level, and one could only be identified at the phylum level.

After filtering of the non-bacterial sequences, the sequences detected in the $P$-negative group were assigned to 294 bacterial OTUs and sorted into 12 phyla, among which Proteobacteria (90.1\%), Firmicutes (6.6\%) and Bacteroidetes $(1.15 \%)$ were the most abundant and accounted for $98 \%$ of the sequence reads. The most abundant bacterial phyla of the 214 bacterial OTUs within the P-positive group were Proteobacteria (80.3\%), Firmicutes (8.7\%), Bacteroidetes (6.7\%) and Actinobacteria (2.7\%) (Fig. 1). Gammaproteobacteria was the predominant class in both groups, but its families had different tendencies. Within the phylum Proteobacteria, Pseudomonadaceae was predominant in the $P$-negative samples, while Enterobacteriaceae was predominant in the $P$-positive samples.

A Venn diagram was employed to display the common and unique bacterial OTUs observed at the genus level in both groups (Additional file 3 ). In the $P$-negative group, 294 bacterial OTUs were detected, while in the $P$-positive group, 214 bacterial OTUs were detected. The proportion of unique bacterial OTUs was $43.54 \%$ in the $P$-negative group and $22.43 \%$ in the $P$-positive group. The percentage of bacterial OTUs detected in both groups was $34.04 \%$.

The genera Pseudomonas and Escherichia/Shigella were the most abundant in the $P$-negative and $P$-positive groups, respectively, compared with the opposite groups. Considering the genera that had relative abundances of more than 1\%, Prevotella 9, Sphingomonas and Bacteroides were found in the P-positive group, and Delftia, Methylobacterium and Bacillus were found in the Pnegative group (Fig. 2). According to the results of previous studies on the microbiota of Anophelinae mosquitoes, the genera Prevotella 9, Sphingomonas, Bacteroides and Bacillus have never been associated with the abdomen of $N y$. darlingi. Composition of the bacterial OTUs from each sample can be viewed in Additional file 4.

\section{PCoA and alpha and beta diversity analyses}

A rarefaction curve was created with a sampling depth of 1500 sequences, showing that the sequencing depth was adequate to infer the structure and abundance of the bacterial community in the abdomen of $N y$. darlingi (Additional file 5). Two sequenced samples (AC141-7 and AC144-17) had sequence numbers lower than the depth used for the rarefaction and therefore were not used in the diversity analyses. To address the bacterial diversity, we used Shannon's diversity index, also known as the Shannon-Weaver index, which considers the 


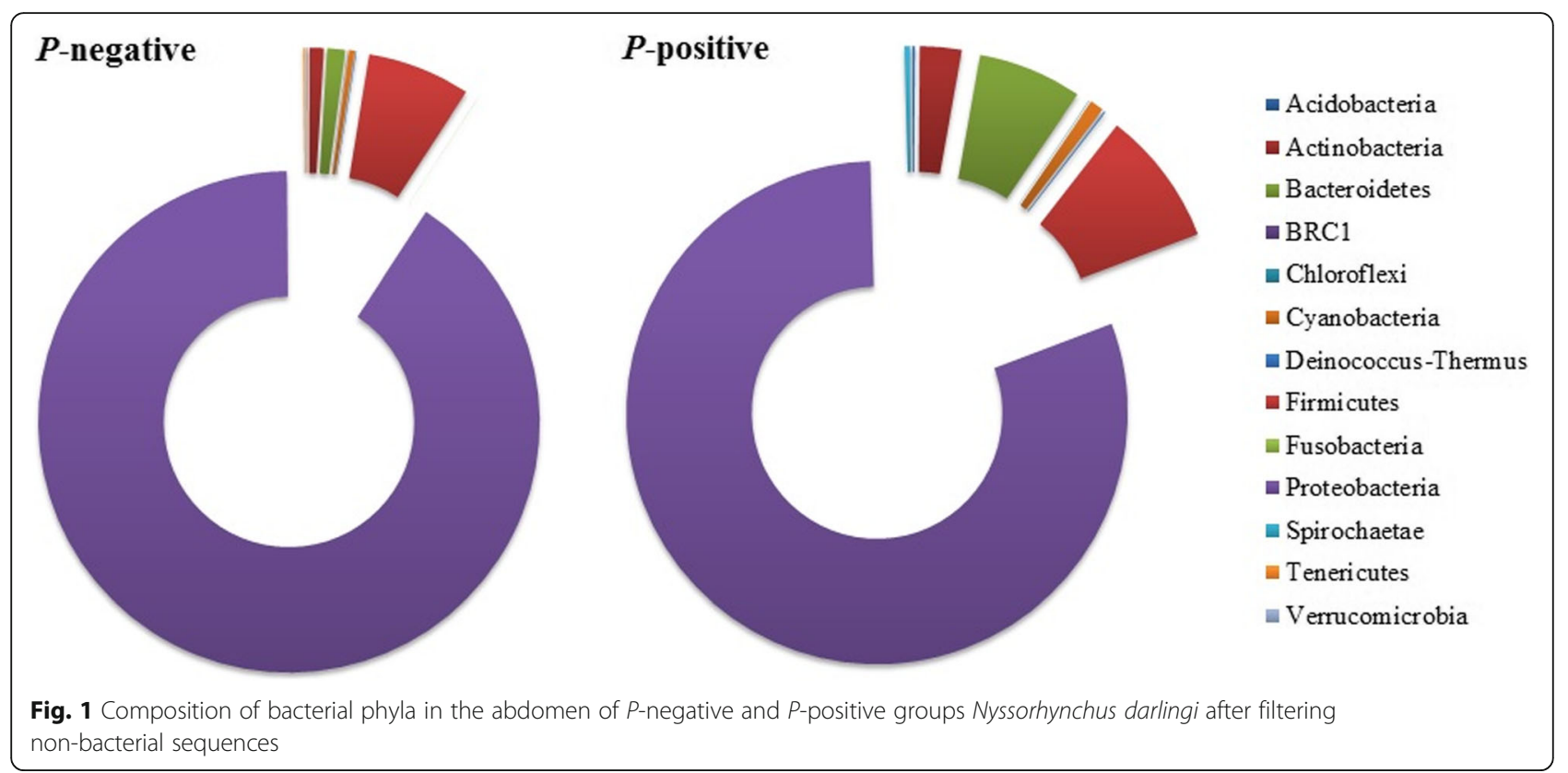

richness and relative abundance of OTUs. The results showed no significant difference between the $P$-negative $(2.78 \pm 0.71)$ and $P$-positive $(2.68 \pm 1.24)$ groups (Additional file 6).

The beta diversity distances between both groups were measured using PCoA. Both PCoA plots of unweighted and weighted UniFrac distances not defined clustering of $P$-negative versus $P$-positive samples (Fig. 3). The PERMANOVA analysis did not detect any difference in the bacterial composition between the $P$-positive and $P$-negative groups $(p>0.05)$. For the above analyses, data from the 407 OTUs generated were used.
Because no significant difference was found in bacterial composition when comparing infected and noninfected groups when samples from all localities were included, beta diversity (PERMANOVA) analysis was performed to compare infected and non-infected groups encompassing females collected in Cruzeiro do Sul and Machadinho D'Oeste separately. The analysis was performed between (1) the infected and noninfected groups (infected $=3$; non-infected $=6$ ) from Cruzeiro do Sul and (2) the infected and non-infected groups (infected $=4$; non-infected $=1$ ) from Machadinho D'Oeste. In both PERMANOVA analyses, there was no significant difference between the groups

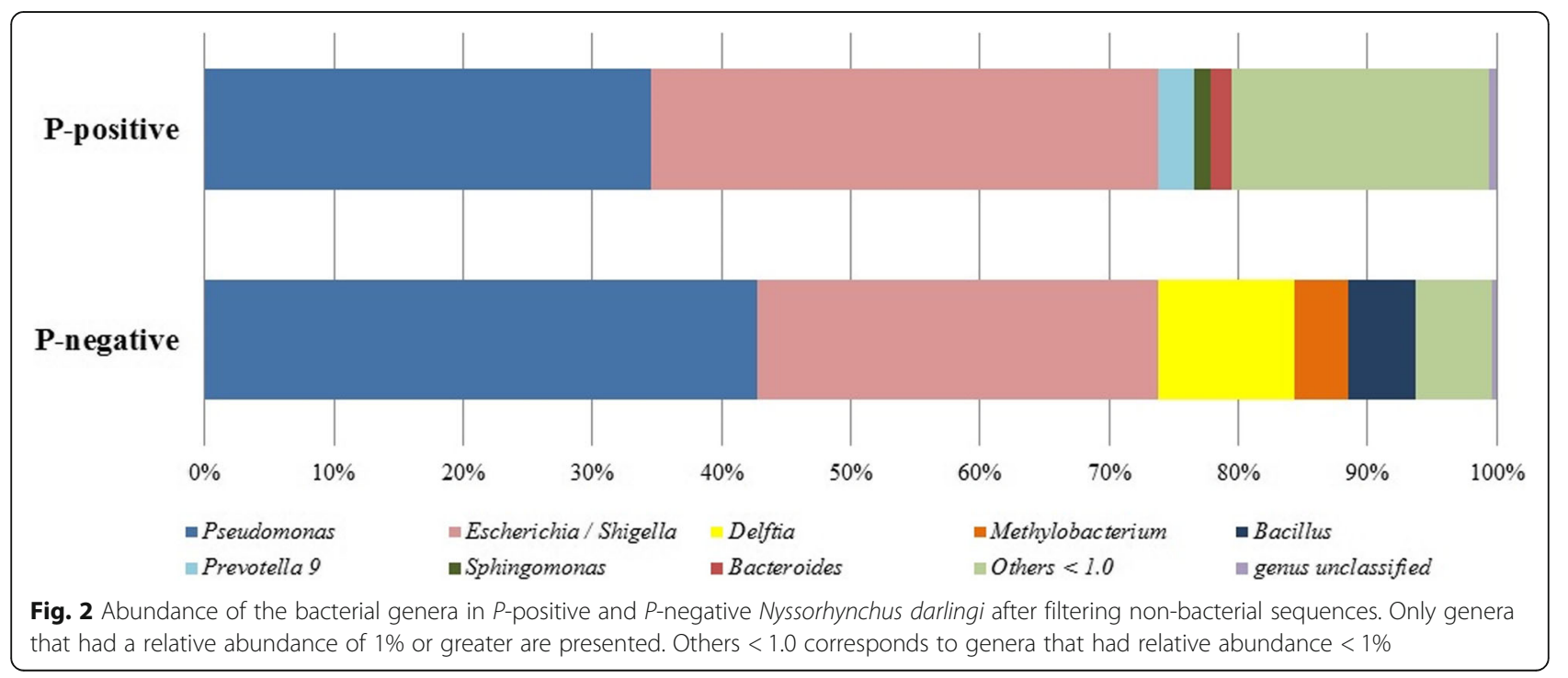




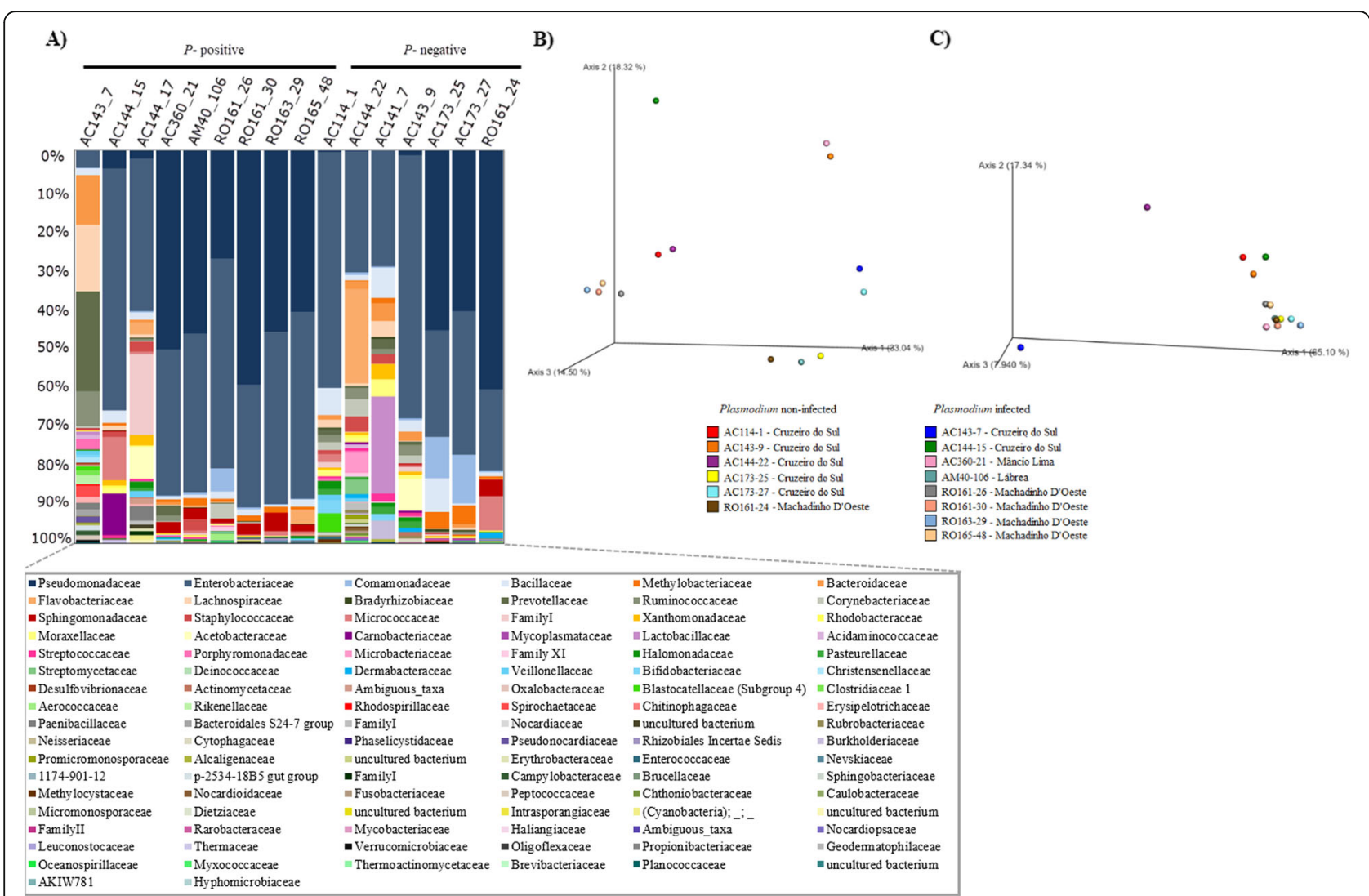

Fig. 3 Beta diversity. Composition of OTUs at family level of all 16 samples (a) and Principal Coordinates Analysis based on the unweighted (b) and weighted (c) UniFrac distances

analysed (Cruzeiro do Sul, $p=0.681$; Machadinho D'Oeste, $p=0.373$ ).

\section{Discussion}

In this study, the bacterial community associated with the abdomen of $N y$. darlingi was investigated. In addition, the bacterial diversity was compared between the two groups studied (the $P$-negative and $P$-positive groups). Next-generation sequencing of PCR products was used to identify the bacterial community associated with the female abdomen. The DNA samples from 7 non-engorged mosquitoes failed to exhibit amplification of $16 \mathrm{~S}$ rRNA after several trials, likely because of the presence of PCR inhibitors or the low numbers of bacteria in these samples. This result is corroborated by those of other studies, such as an investigation conducted by Terenius et al. [14], in which only one of four non-blood-fed $N y$. darlingi presented amplification of the16S rRNA gene.

The bacteria in adult $N y$. darlingi abdomens comprised three predominant phyla: Proteobacteria, Firmicutes and Bacteroidetes. This result presents similarity to the results of other studies performed with Anophelinae mosquitoes $[17,18]$ and suggests that bacteria of these phyla have importance in the physiology of Anophelinae mosquitoes. The number of OTUs identified was higher than those found in other conventional molecular studies [14]. Of the 379 bacterial OTUs, only 7 showed an abundance higher than $2 \%$, suggesting that there are few predominant bacterial genera. Minard et al. [19] reported that mosquito females are colonized mainly by Gammaproteobacteria. Similarly, Gammaproteobacteria was the most prevalent class of bacteria identified in the females analysed (16/16 samples). Gammaproteobacteria includes the Enterobacteriaceae and Pseudomonadaceae families.

Enterobacteriaceae has been found in abundance in Plasmodium-infected An. gambiae, indicating that bacteria of this family contribute to the development of the parasite [20]. Although Enterobacteriaceae was the most abundant family identified in the $N y$. darlingi P-positive group, there was no significant difference in diversity between the $P$-positive and $P$-negative groups $(p>0.05)$. Inconsistent results of studies can be caused by various factors, including differences in experimental design and sample preparation. For example, the females used by Boissière et al. [20] were fed $P$. falciparum-infected blood, and the midgut microbiota was analysed 8 days 
after the blood meal. In our study, field-collected females were found naturally infected with Plasmodium, but we did not have information about the time of the mosquito blood meal. Considering that the females were naturally infected, they would have fed on Plasmodium-infected human blood at least 10-15 days before [21]. The differences observed may be associated with the time of the infected blood meal and the HLC collection procedure.

Anopheles gambiae exposed to an Enterobacter (Esp_ $Z$ ) isolate have been found to become less susceptible to P. falciparum infection [3]. Enterobacter was recovered from $2 N y$. darlingi females, neither of which was infected with Plasmodium. Despite this finding, it is premature to hypothesize that females with Enterobacter are less susceptible to $P$. falciparum infection than those without it, because our sample was small and limited to a few localities with moderate, low or moderately high transmission [22]. These two limitations prevent us from drawing any conclusions about the impact of Enterobacter on vector species susceptibility to $P$. falciparum infection. Further studies will be necessary to verify whether any Enterobacter isolates can decrease $N y$. darlingi susceptibility to Plasmodium infection and to determine if the inhibition mechanism is similar to that of the $E s p_{-} Z$ isolate in An. gambiae, which is mediated by the generation of reactive oxygen species (ROS).

Pseudomonas was found in $N y$. darlingi and has been widely reported in anopheline mosquitoes from Africa, Asia and America $[14,15,23-25]$. In this study, Pseudomonas was the most abundant genus in the $P$-negative group and was detected in 15 out of 16 samples analysed. These data corroborate those of a study by Chavshin et al. [26], who reported Pseudomonas as the most common bacterial isolate in Anopheles stephensi larvae and adults, indicating possible transmission of Pseudomonas between mosquito developmental stages. The high prevalence of Pseudomonas in Anopheles mosquitoes suggests the capability of Pseudomonas to easily adapt to the midgut environment.

The most predominant bacteria in the $P$-positive group detected in all investigated samples were found to belong to the genus Escherichia/Shigella. In previous studies, this genus has been found in all mosquito samples analysed in Northern California (USA) [27] and in more than $85 \%$ of samples of An. gambiae [20]. Both studies have also reported the prevalence of this genus in other species of mosquitoes. The fact that these bacteria were found in $N y$. darlingi breeding sites located in Manaus in the Brazilian Amazon region [28] suggests that this genus is either acquired during immature development or immediately after emergence.

Although the $P$-negative group had a higher number of OTUs than the $P$-positive group, there was no significant difference in bacterial diversity between the groups
( $P$-positive and $P$-negative); neither group was more diverse than the other. Additionally, there was no significant difference in the composition of the bacteria between the groups (beta diversity), not even between the infected and non-infected groups of the same municipality (Cruzeiro do Sul and Machadinho D'Oeste). These results appear contrary to the findings of Bassene et al. [29], who reported greater bacterial diversity in vectors (An. gambiae and An. funestus) infected with P. falciparum. Possibly, this difference in results could be related to the fact that we did not differentiate whether the $N y$. darlingi infection was caused by $P$. vivax or $P$. falciparum.

Another possible explanation for the lack of statistical significance difference, not addressed here, could be due to the effects of $N y$. darlingi genotype variant on microbial communities [30-32]. Several studies have shown that various factors such as nutrition, sex, environment, and genotype can all shape the pattern of mosquitoesassociated microbiota [19, 33]. Despite these reports, Minard et al. [33] demonstrated that the Aedes albopictus genetic variation is correlated with microbiome composition. However, in another study with populations of Ae. albopictus reared in the laboratory, Minard et al. [34] verified that when environmental factors were removed, there were no differences in the microbiota diversity between the populations studied. Dickson et al. [35] found that the microbiota diversity in the Aedes aegypti is determined by the environment, regardless of the host genotype. Thus, further studies are needed to verify whether different genotypes of $N y$. darlingi can influence the bacterial composition of the microbiota.

Considering that our study employed field collected females, the number of specimens analyzed was small. Further studies will be needed to test the hypotheses and address if the load of Plasmodium parasites is associated with the divergence in the mosquito gut microbiota.

\section{Conclusions}

Here, we provide data on bacterial communities associated with the abdomens of $N y$. darlingi naturally infected and not infected with Plasmodium. The genera Prevotella 9, Sphingomonas, Bacteroides and Bacillus are reported for the first time in $N y$. darlingi. Our data contribute to a better understanding of the bacteriome composition in Ny. darlingi. Since Enterobacter was found in two non-infected $N y$. darlingi samples and was absent in the $P$-positive group, other studies are necessary to determine if Enterobacter species present in Ny. darlingi offer protection against Plasmodium infection.

\section{Methods}

\section{Mosquito collections}

Females of $N y$. darlingi were collected in the municipalities of Cruzeiro do Sul (Acre state), Mâncio Lima (Acre 
state), Lábrea (Amazonas state), and Machadinho D'Oeste (Rondônia state), Brazilian Amazon (Additional file 1). Field collections were conducted in peridomestic habitats by human landing catches (HLCs). Mosquitoes were killed with ethyl acetate $\left(\mathrm{C}_{4} \mathrm{H}_{8} \mathrm{O}_{2}\right)$ and immediately preserved in silica gel until species identification. Mosquito species were identified using morphological characteristics. Following identification, females were preserved at $-80^{\circ} \mathrm{C}$.

\section{Plasmodium testing}

Each $N y$. darlingi female collected was divided into the head/thorax and abdomen. The DNA of the head/thorax was extracted according to Laporta et al. [36] and used for Plasmodium testing. Plasmodium detection was performed by using real-time PCR of the $18 \mathrm{~S}$ rRNA region followed by high-resolution melting (HRM) analysis. For each $20 \mu \mathrm{l}$ reaction, $1 \mathrm{X}$ MeltDoctor ${ }^{\mathrm{Tm}}$ HRM Master Mix (ThermoFisher Scientific, Inc., Waltham, MA, USA), 10 ng of DNA, $500 \mathrm{nM}$ of each primer (1459-M13[forward, 5'TGTAAAACGACGGCCAGTCTGGTTAATTCCGATAAC 3'] and 1706-M13 [reverse, 5'CAGGAAACAGCTATGACCTAAACTTCCTTGTGTTAGAC 3']) [37] and commercial ultrapure $\mathrm{H}_{2} \mathrm{O}$ were added. The cycling program consisted of $95^{\circ} \mathrm{C}$ for $10 \mathrm{~min}$ followed by $50 \mathrm{cy}$ cles of $95^{\circ} \mathrm{C}$ for $15 \mathrm{~s}$ and $60^{\circ} \mathrm{C}$ for $1 \mathrm{~min}$. The reaction was then followed by HRM curve analysis, which was conducted by increasing the temperature from $60^{\circ} \mathrm{C}$ to $95^{\circ} \mathrm{C}$ with a ramp rate of $0.3 \%$. The results were analysed using StepOnePlus ${ }^{\text {ti }}$ Software v2.3 and HRM v3.0.1 Software. Seventy samples were infected with Plasmodium. The corresponding abdomens from 13 infected samples and 11 Plasmodium-uninfected female $N y$. darlingi were used for the $16 \mathrm{~S}$ rRNA amplicon survey (Additional file 1).

\section{Sequencing of the V4 region of 16S rRNA}

Abdomens of $N y$. darlingi were surface rinsed twice in $70 \%$ ethanol and ultrapure water. The genomic DNA of each individual abdomen was extracted by using a DNeasy PowerSoil kit (Qiagen, Hilden, Germany) or by the salt precipitation method [36]. For the DNeasy PowerSoil Kit, each abdomen was placed in PowerBead tubes (included in the PowerSoil kit) and incubated at room temperature for $15 \mathrm{~min}$ before bead beating for another $15 \mathrm{~min}$. After these steps, genomic DNA was extracted according to the manufacturer's instructions. For the salt precipitation method, each abdomen was mixed in $500 \mu \mathrm{L}$ of TEN buffer $(2 \mathrm{mM}$ Tris- $\mathrm{HCl} \mathrm{pH} 8.0,0.5$ $\mathrm{mM}$ EDTA, $5 \mathrm{mM} \mathrm{NaCl}$ ) and homogenized using 1mm-diameter zirconia beads (Biospec, Bartlesville, USA) via the use of BeadBlaster24 equipment (Benchmark Scientific, Inc., Sayreville, NJ, USA) for 4 cycles with shaking $(4 \mathrm{~m} / \mathrm{sec})$ for $40 \mathrm{~s}$ followed by $20 \mathrm{~s}$ without shaking in each cycle. A lysis buffer containing $5 \mu \mathrm{L}$ of $10 \%$ SDS (Promega, Madison, USA) and $3 \mu \mathrm{L}$ of $20 \mathrm{mg} / \mathrm{mL}$ proteinase $\mathrm{K}$ (Promega) was added to the homogenate and mixed. After $1 \mathrm{~h}$ of incubation at $56^{\circ} \mathrm{C}, 150 \mu \mathrm{L}$ of $5 \mathrm{M}$ $\mathrm{NaCl}$ was added, and the mixture was shaken vigorously for $15 \mathrm{~s}$. The mixture was centrifuged for $10 \mathrm{~min}$ at 13 , $000 \mathrm{rpm}$ at $20^{\circ} \mathrm{C}$, and the supernatant was gently transferred into a new tube. Immediately, $600 \mu \mathrm{L}$ of freshly prepared cold isopropyl alcohol was added to the supernatant and mixed by inversion, and the mixture was incubated at $-20^{\circ} \mathrm{C}$ for $48 \mathrm{~h}$. The supernatant was decanted after a $10 \mathrm{~min}$ centrifugation. The remaining pellet was washed with $1 \mathrm{~mL}$ of $70 \%$ ethanol, and the dried pellet was resuspended in $20 \mu \mathrm{L}$ of TE buffer ( 2 $\mathrm{mM}$ Tris- $\mathrm{HCl}, \mathrm{pH}$ 8.0, 0.5 mM EDTA).

The V4 hypervariable region of the 16S rRNA gene was amplified according to Caporaso et al. [38]. Briefly, each reaction was performed in a final volume of $20 \mu \mathrm{L}$ consisting of $1 \mathrm{X} \mathrm{GoTaq}{ }^{\circ}$ Colorless Master Mix (Promega), $0.3 \mu \mathrm{M}$ of each primer (Additional file 7), $2 \mu \mathrm{L}$ of genomic DNA and ultrapure water. The thermocycling conditions were $94^{\circ} \mathrm{C}$ for $3 \mathrm{~min}$ followed by 29 cycles of $94{ }^{\circ} \mathrm{C}$ for $45 \mathrm{~s}, 50^{\circ} \mathrm{C}$ for $1 \mathrm{~min}, 72^{\circ} \mathrm{C}$ for $1 \mathrm{~min}$ and $30 \mathrm{~s}$, and a final extension of $72^{\circ} \mathrm{C}$ for $10 \mathrm{~min}$. The reactions were carried out in triplicate. The PCR products were visualized on a $2 \%$ agarose gel stained with Gelred ${ }^{\circ}$ (Uniscience, Miami, USA). The PCR products were purified with Agencourt AMPure XP magnetic beads (Beckman Coulter, Brea, USA) and then quantified by real-time PCR with KAPA (KAPA Biosystems, Wilmington, USA) according to the manufacturer's recommendations.

All samples were normalized to $3 \mathrm{nM}$, and an equimolar pool of DNA was prepared. Next-generation sequencing of the V4 region was performed on an Illumina MiSeq sequencer (Illumina, San Diego, USA) using a MiSeq Reagent Micro v2 kit (300 cycles).

\section{Processing of sequences and taxonomic attribution} Illumina paired-end reads were assembled in QIIME v.1.9 [39] with a minimum overlap of 20 base pairs (join_paired_ends.py). The sequences were filtered based on the length and sequencing error rates $\left(E_{-} \max =1\right)$. USEARCH v.11 [40, 41] was used to filter sequences (fastq_filter), discard singletons (sortbysize), and remove chimaeras and cluster sequences in unique OTUs (cluster_otus). The taxonomic classification of each read was assigned against Silva v.128 [42] at a 99\% threshold of pairwise sequence similarity using QIIME v.1.9. All OTUs that had fewer than 5 sequences were removed from the analysis.

\section{Diversity index determination and statistical analysis}

The Shannon-Weaver index $(\mathrm{H})$ and observed OTU index $(\mathrm{S})$ were used to describe the bacterial diversity 
(alpha diversity) of each separate specimen and each group ( $P$-positive and $P$-negative). The Wilcoxon-MannWhitney test was used to compare the results for both groups. Dissimilarity analyses (beta-diversity) between the $P$-positive and $P$-negative groups were performed using the unweighted and weighted UniFrac distances and a sampling depth of 1500 sequences. The unweighted UniFrac distance is a qualitative measure that uses phylogenetic information to compare biological communities, while the weighted Unifrac distance is a quantitative measure. Principal coordinate analyses (PCoA) of the unweighted and weighted UniFrac distances of bacterial communities were conducted to measure the distance between communities. Permutational multivariate analysis of variance (PERMANOVA) was employed to measure intergroup distances. The PCoA and PERMANOVA analyses were conducted with rarefied data recovered after rarefying steps in QIIME2 (2019.1 version)

\section{Supplementary information}

Supplementary information accompanies this paper at https://doi.org/10 1186/s12866-020-01861-0.

Additional file 1. ID sample, Municipality of collection and Plasmodium infection of each sample used in this study

Additional file 2. Number of OTUs sequences from each sample, after removing OTUs with less than 5 sequences.

Additional file 3. Venn diagram display the number of unique bacterial OTUs in $P$-negative and $P$-positive $N y$. darlingi. Number of OTUs after filtering non-bacterial sequences.

Additional file 4. Composition of abundant OTUs at family level of all 16 samples after filtering non-bacterial sequences. Samples collected in Cruzeiro do Sul: AC114-1, AC141-7, AC143-9, AC173-25, AC173-27, AC143-7, AC144-22, AC144-15 and AC144-17. Sample collected in Mâncio Lima: AC360-21. Sample collected in Lábrea: AM40-106. Samples collected in Machadinho D'Oeste: RO161-24, RO161-26, RO161-30, RO16329 and RO165-48.

Additional file 5. Rarefaction curves of OTUs ( $97 \%$ similarity) of V4 region of $16 \mathrm{~S}$ rRNA gene sequences from 16 samples. Rarefaction curves were generated in Qiime2 (2019.1 version). Bars correspond to the standard deviation in each depth step after 10 iterations (rarefied tables computed at each sampling depth).

Additional file 6 Box plot of Shannon index for $P$-negative and $P$ positive groups. Center lines show the medians; box limits indicate the 25th and 75th percentiles; whiskers extend to 5th and 95th percentiles, outliers are represented by dots.

Additional file 7. Sequence of the oligonucleotides used to amplify the $\checkmark 4$ region of the 165 rRNA gene.

\section{Abbreviations}

DNA: Deoxyribonucleic Acid; EDTA: Ethylenediamine Tetraacetic Acid; HCL: Hydrochloric Acid; HLC: Human Landing Catch; HRM: High-Resolution Melting; NaCl: Sodium Chloride; OTU: Operational Taxonomic Unit; PCoA: Principal Coordinates Analysis; PCR: Polymerase Chain Reaction; PERMANOVA: Permutational Multivariate Analysis of Variance; QIIME: Quantitative Insights Into Microbial Ecology; rRNA: Ribosomal Ribonucleic Acid; ROS: Reactive Oxygen Species; SDS: Sodium Dodecyl Sulfate; TE: Tris-EDTA; WHO: World Health Organization

\section{Acknowledgments}

We are in debt to Eduardo Sterlino Bergo, Gabriel Zorello Laporta and Leonardo Suveges Moreira Chaves for their valuable contribution to field collections and for two anonymous reviewers for their contribution to improve the manuscript.

\section{Authors' contributions}

TMPO and MAMS conceived the experiments. MAMS planned and conducted the field collections. MAMS identified field specimens. TMPO and SS conducted bioinformatics analyses. TMPO conducted the laboratory experiments. TMPO, MAMS and SS wrote the manuscript. All authors have read and approved the manuscript.

\section{Funding}

This work has been funded by the Fundação de Amparo à Pesquisa do Estado de São Paulo - FAPESP, Grant no. 2014/26229-7, and Conselho Nacional de Pesquisa - CNPq no. 301877/2016-5 to MAMS

\section{Availability of data and materials}

The datasets generated and analysed during the current study are available in the European Nucleotide Archive (ENA) repository (Project: PRJEB32570, Access numbers: ERR3324411-ERR3324396). The sequences of the 407 OTUs are available in the Zenodo repository (https://doi.org/10.5281/zenodo. 3738681).

\section{Ethics approval and consent to participate}

All necessary permits were obtained for the described study. Mosquito collections were carried out under permit no. 16938-1 from Instituto Brasileiro do Meio Ambiente e dos Recursos Naturais Renováveis (IBAMA) to M.A.S.M. Specific permission was not required for these locations as permission to collect was granted under the permanent permit. The collection locations were not privately owned or protected in any way, and the field collections did not involve protected or endangered species.

\section{Consent for publication}

Not applicable.

\section{Competing interests}

The authors declare that they have no competing interests.

\section{Author details}

'Departamento de Epidemiologia, Faculdade de Saúde Pública, Universidade de São Paulo, São Paulo, SP, Brazil. 'LIM-3, Hospital das Clínicas da FMUSP (HCFMUSP), Faculty of Medicine, University of São Paulo, São Paulo, Brazil.

Received: 4 February 2020 Accepted: 16 June 2020

Published online: 25 June 2020

References

1. World Health Organization. World Malaria Report 2018. http://www.who. int/malaria/publications/world-malaria-report-2018/report/en/. Accessed 27 Jan 2019

2. Ferreira MU, Castro MC. Challenges for malaria elimination in Brazil. Malar J. 2016. https://doi.org/10.1186/s12936-016-1335-1.

3. Cirimotich CM, Dong Y, Clayton AM, Sandiford SL, Souza-Neto JA, Mulenga $M$, et al. Natural microbe-mediated refractoriness to Plasmodium infection in Anopheles gambiae. Science. 2011;332(6031):855-8.

4. Bando H, Okado K, Guelbeogo WM, Badolo A, Aonuma H, Nelson B, et al. Intra-specific diversity of Serratia marcescens in Anopheles mosquito midqut defines Plasmodium transmission capacity. Sci Rep. 2013. https://doi.org/10. 1038/srep01641.

5. Tchioffo MT, Boissière A, Churcher TS, Abate L, Gimonneau G, Nsango SE, Awono-Ambéne PH, Christen R, Berry A, Morlais I. Modulation of malaria infection in Anopheles gambiae mosquitoes exposed to natural Midgut Bacteria. PLoS One. 2013. https://doi.org/10.1371/journal.pone.0081663.

6. Gendrin M, Rodgers FH, Yerbanga RS, Ouédraogo JB, Basáñez MG Cohuet A, Christophides GK. Antibiotics in ingested human blood affect the mosquito microbiota and capacity to transmit malaria. Nat Commun. 2015;6:1-7.

7. Baldini F, Rougé J, Kreppel K, Mkandawile G, Mapua SA Sikulu-Lord M, et al. First report of natural Wolbachia infection in the malaria mosquito 
Anopheles arabiensis in Tanzania. Parasit Vectors. 2018. https://doi.org/10. 1186/s13071-018-3249-y.

8. Kalappa DM, Subramani PA, Basavanna SK, Ghosh SK, Sundarrramurthy V, Uragayala S, Tiwari S, Anvikar A, Valecha N. Influence of midgut microbiota in Anopheles stephensi on Plasmodium berghei infections. Malar J. 2018. https://doi.org/10.1186/s12936-018-2535-7.

9. Gonzalez-Ceron L, Santillan F, Rodriguez MH, Mendez D, Hernandez-Avila JE. Bacterial in midguts of field-collected Anopheles albimanus block Plasmodium vivax sporogonic development. J Med Entomol. 2003;40(3):371-4.

10. Bahia AC, Dong Y, Blumberg BJ, Mlambo G, Tripathi A, Benmarzouk-Hidalgo OJ, et al. Exploring Anopheles gut bacteria for Plasmodium blocking activity. Environ Microbiol. 2014;16(9):2980-94.

11. Dong Y, Manfredini F, Dimopoulos G. Implication of the mosquito Midgut microbiota in the defense against malaria parasites. PLoS Pathog. 2009;5:1-10.

12. Romoli O, Gendrin M. The tripartite interactions between the mosquito, its microbiota and Plasmodium. Parasit Vectors. 2018. https://doi.org/10.1186/ s13071-018-2784-X

13. Beier MS, Pumpuni CB, Beier JC, Davis JR. Effects of Para-aminobenzoic acid, insulin, and gentamicin on Plasmodium falciparum development in anopheline mosquitoes (Diptera: Culicidae). J Med Entomol. 1994;31(4):561-5.

14. Terenius O, Oliveira CD, Pinheiro WD, Tadei WP, James AA, Marinotti O. $16 \mathrm{~S}$ rRNA gene sequences from Bacteria associated with adult Anopheles darlingi (Diptera: Culicidae) mosquitoes. J Med Entomol. 2008:45(1):172-5.

15. Bascuñán P, Niño-Garcia JP, Galeano-Castañeda Y, Serre D, Correa MM. Factors shaping the gut bacterial community assembly in two main Colombian malaria vectors. Microbiome. 2018. https://doi.org/10.1186/ s40168-018-0528-y.

16. Prussing C, Saavedra MP, Bickersmith SA, Alava F, Guzmán M, Manrique E, Carrasco-Escobar G, Moreno M, Gamboa D, Vinetz JM, Conn JE. Malaria vector species in Amazonian Peru co-occur in larval habitats but have distinct larval microbial communities. PLoS Negl Trop Dis. 2019;13(5): e0007412.

17. Ngo CT, Romano-Bertrand S, Manguin S, Jumas-Bilak E. Diversity of the bacterial microbiota of Anopheles mosquitoes from Binh Phuoc Province, Vietnam. Front Microbiol. 2016. https://doi.org/10.3389/fmicb.2016.02095

18. Tchioffo MT, Boissière A, Abate L, Nsango SE, Bayibéki AN, Awono-Amnéne $\mathrm{PH}$, et al. Dynamics of bacterial community composition in the malaria mosquito's epithelia. Front Microbiol. 2016. https://doi.org/10.3389/fmicb. 2015.01500.

19. Minard G, Mavingui P, Moro CV. Diversity and function of bacteria microbiota in the mosquito holobiont. Parasit Vectors. 2013. https://doi.org/ 10.1186/1756-3305-6-146..

20. Boissière A, Tchioffo MT, Bachar D, Abate L, Marie A, Nsango SE, et al. Midgut microbiota of the malaria mosquito vector Anopheles gambiae and interactions with Plasmodium falciparum infection. PLoS Pathog. 2012. https://doi.org/10.1371/journal.ppat.1002742.

21. Smith RC, Vega-Rodríguez J, Jacobs-Lorena M. The Plasmodium bottleneck: malaria parasite losses in the mosquito vector. Mem Inst Oswaldo Cruz. 2014:109:644-61

22. Sallum MAM, Conn JE, Bergo ES, Laporta GZ, Chaves LSM, Bickersmith SA, de Oliveira TMP, Figueira EAG, Moresco G, Olívêr L, Struchiner CJ, Yakob L, Massad E. Vector competence, vectorial capacity of Nyssorhynchus darlingi and the basic reproduction number of Plasmodium vivax in agricultural settlements in the Amazonian region of Brazil. Malar J. 2019;18(1):117.

23. Rani A, Sharma A, Rajagopal R, Adak T, Bhatnagar RK. Bacterial diversity analysis of larvae and adult midgut microflora using culture-dependent and culture-independent methods in lab-reared and field-collected Anopheles stephensi - an Asian malarial vector. BMC Microbiol. 2009. https://doi.org/10. 1186/1471-2180-9-96.

24. Chavshin AR, Oshaghi MA, Vatandoost H, Pourmand MR, Raeisi A, Terenius O. Isolation and identification of culturable bacteria from wild Anopheles culicifacies, a first step in a paratransgenesis approach. Parasit Vectors. 2014. https://doi.org/10.1186/1756-3305-7-419.

25. Raharimalala FN, Bokraa S, Bawin T, Boyer S, Francis F. Molecular detection of six (endo-) symbiotic bacteria in Belgian mosquitoes: first step towards the selection of appropriate paratransgenesis candidates. Parasitol Res. 2016; 115(4):1391-9.

26. Chavshin AR, Oshaghi MA, Vatandoost $H$, Pourmand MR, Raeisi A, Enayati $\mathrm{AA}$, et al. Identification of bacterial microflora in the midgut of the larvae and adult of wild caught Anopheles stephensi: a step toward finding suitable paratransgenesis candidates. Acta Trop. 2012;121 (2):129-34.
27. Chandler JA, Liu RM, Bennett SN. RNA shotgun metagenomic sequencing of northern California (USA) mosquitoes uncovers viruses, bacteria, and fungi. Front Microbiol. 2015. https://doi.org/10.3389/fmicb.2015.00185.

28. Nilsson LK, Oliveira MR, Marinotti O, Rocha EM, Håkansson S, Tadei WP, Souza AQL, Terenius O. Characterization of bacterial communities in breeding waters of Anopheles darlingi in Manaus in the Amazon Basin malaria-endemic area. Microb Ecol. 2019;78:781-91.

29. Bassene H, Niang EHA, Fenollar F, Dipankar B, Doucouré S, Ali E, et al. 165 metagenomic Comparasion of Plasmodium falciparum-infected and noninfected Anopheles gambiae and Anopheles funestus microbiota from Senegal. Am J Trop Med Hyg. 2018;99(6):1489-98.

30. Angêlla AF, Salgueiro P, Gil LHS, Vicente JL, Pinto J, Ribolla PEM. Seasonal genetic partitioning in the neotropical malaria vector, Anopheles darlingi. Malar J. 2014. https://doi.org/10.1186/1475-2875-13-203.

31. Campos M, Conn JE, Alonso DP, Vinetz JM, Emerson KJ, Ribolla PEM. Microgeographical structure in the major Neotropical malaria vector Anopheles darlingi using microsatellites and SNP markers. Parasit Vectors. 2017. https://doi.org/10.1186/s13071-017-2014-y.

32. Prussing C, Emerson KJ, Bickersmith SA, Sallum MAM, Conn JE. Minimal genetic differentiation of the malaria vector Nyssorhynchus darlingi associated with forest cover level in Amazonian Brazil. PLoS One. 2019. https://doi.org/10.1371/journal.pone.0225005.

33. Minard G, Tran FH, Van VT, Goubert C, Bellet C, Lambert G, et al. French invasive Asian tiger mosquito populations harbor reduced bacterial microbiota and genetic diversity compared to Vietnamese autochthonous relatives. Front Microbiol. 2015. https://doi.org/10.3389/fmicb.2015.00970.

34. Minard G, Tran FH, Tran Van V, Fournier C, Potier P, Roiz D, Mavingui P, Valiente Moro C. Shared larval rearing environment, sex, female size and genetic diversity shape Ae. albopictus bacterial microbiota. PLoS One. 2018; doi: https://doi.org/10.1371/journal.pone.0194521.

35. Dickson LB, Ghozlane A, Volant S, Bouchier C, Ma L, Vega-Rúa A, Dusfour I, Jiolle D, Paupy C, Mayanja MN, Kohl A, Lutwama JJ, Duong V, Lambrechts L. Diverse laboratory colonies of Aedes aegypti harbor the same adult midgut bacterial microbiome. Parasit Vectors. 2018. https://doi.org/10.1186/s13071018-2780-1.

36. Laporta GZ, Burattini MN, Levy D, Fukuya LA, Oliveira TMP, Maselli LMF, et al. Plasmodium falciparum in the southeastern Atlantic forest: a challenge to the bromeliad-malaria paradigm? Malar J. 2015. https://doi.org/10.1186/ s12936-015-0680-9.

37. Kipanga PN, Omondi D, Mireji PO, Sawa P, Masiga DK, Villinger J. Highresolution melting analysis reveals low Plasmodium parasitaemia infections among microscopically negative febrile patients in western Kenya. Malar J. 2014. https://doi.org/10.1186/1475-2875-13-429.

38. Caporaso JG, Lauber CL, Walters WA, Berg-Lyons D, Lozupone CA, Turnbaugh PJ. Global patterns of 165 rRNA diversity at a depth of millions of sequences per sample. Proc Natl Acad Sci U S A. 2011;108(Suppl 1):4516-22.

39. Caporaso JG, Kucznski J, Stombaugh J, Bittinger K, Bushman FD, Costello EK. QIIME allows analysis of high-throughput community sequencing data. Nat Methods. 2010;7(5):335-6.

40. Edgar RC. Search and clustering orders of magnitude faster than BLAST. Bioinformatics. 2010;26(19):2460-1.

41. Edgar RC. UPARSE: highly accurate OTU sequences from microbial amplicon reads. Nat Methods. 2013;10(10):996-8.

42. Pruesse E, Quast C, Knittel K, Fuchs BM, Ludwig WG, Peplies J, et al. SILVA: a comprehensive online resource for quality checked and aligned ribosomal RNA sequence data compatible with ARB. Nucleic Acids Res. 2007;35(21): $7188-96$.

\section{Publisher's Note}

Springer Nature remains neutral with regard to jurisdictional claims in published maps and institutional affiliations. 